\title{
Acoustic oscillations in a field-free cavity under solar small-scale bipolar magnetic canopy
}

\author{
D. Kuridze ${ }^{1}$, T. V. Zaqarashvili ${ }^{1}$, B. M. Shergelashvili ${ }^{1,2}$, and S. Poedts ${ }^{3}$ \\ ${ }^{1}$ Georgian National Astrophysical Observatory (Abastumani Astrophysical Observatory), Al. Kazbegi ave. 2a, 0160 Tbilisi, \\ Georgia \\ ${ }^{2}$ Institute for Theoretical Physics, K.U. Leuven, Celestijnenlaan 200 D, 3001, Leuven, Belgium \\ ${ }^{3}$ Center for Plasma Astrophysics, K.U.Leuven, 200 B, 3001, Leuven, Belgium
}

Received: 9 October 2007 - Revised: 16 January 2008 - Accepted: 16 January 2008 - Published: 15 October 2008

\begin{abstract}
Observations show the increase of high-frequency wave power near magnetic network cores and active regions in the solar lower atmosphere. This phenomenon can be explained by the interaction of acoustic waves with a magnetic field. We consider small-scale, bipolar, magnetic field canopy structure near the network cores and active regions overlying field-free cylindrical cavities of the photosphere. Solving the plasma equations we get the analytical dispersion relation of acoustic oscillations in the field-free cavity area. We found that the $m=1$ mode, where $m$ is azimuthal wave number, cannot be trapped under the canopy due to energy leakage upwards. However, higher $(m \geq 2)$ harmonics can be easily trapped leading to the observed acoustic power halos under the canopy.
\end{abstract}

Keywords. Ionosphere (Wave propagation) - Solar physics, astrophysics, and astronomy (Photosphere and chromosphere)

\section{Introduction}

Waves play an important role in the dynamics of the solar atmosphere. Observations show an increase of high-frequency power $(v>5 \mathrm{mHz})$ in the surroundings of active regions in velocity power maps sometimes called as photospheric power halos (Braun et al., 1992; Brown et al., 1992; Hindman and Brown, 1998; Jain and Haber, 2002). The halos were not found in Doppler power maps at lower frequencies $(3 \mathrm{mHz})$. Observations also show a lack of power halos in continuum

Correspondence to: D. Kuridze

(dato.k@genao.org) intensity power maps (Hindman and Brown, 1998; Jain and Haber, 2002; Muglach et al., 2005).

On the other hand, recent observations reveal the decrease of the acoustic high frequency power in the chromosphere and its increase in the photosphere near active regions (Muglach, 2003; Muglach et al., 2005). It has also been shown that the quiet-Sun chromospheric magnetic network elements are surrounded by "magnetic shadows", which lack the oscillatory power at higher frequency range (McIntosh and Judge, 2001; Krijger et al., 2001; Vecchio et al., 2007). Therefore, both the photospheric power halos and the chromospheric magnetic shadows probably reflect the same physical process of acoustic wave interaction with overlying magnetic field (while in subsurface regions the rotational and the meridional non-uniform flows are supposed to have an impact on the formation of the acoustic wave power spectra. Shergelashvili and Poedts, 2005).

The properties of propagating acoustic waves are closely related to the magnetic field structure. The numerical calculations show that the propagation of acoustic disturbances in the solar atmosphere is strongly determined by the overlying magnetic canopy (Rosenthal et al., 2002; Bogdan et al., 2003). The canopy has been usually modeled with purely horizontal magnetic field (Evans and Roberts, 1990), but recent high-resolution observations reveal more complex small-scale structure of the field (De Wijn et al., 2005; Centeno et al., 2007). It has been suggested that the magnetic field has small-scale closed loop structure in the vicinity of network cores (McIntosh and Judge, 2001; Schrijver and Title, 2003). The inclined magnetic field may channel low-frequency photospheric oscillations in the chromosphere/corona (De Pontieu et al., 2004).

Published by Copernicus Publications on behalf of the European Geosciences Union. 


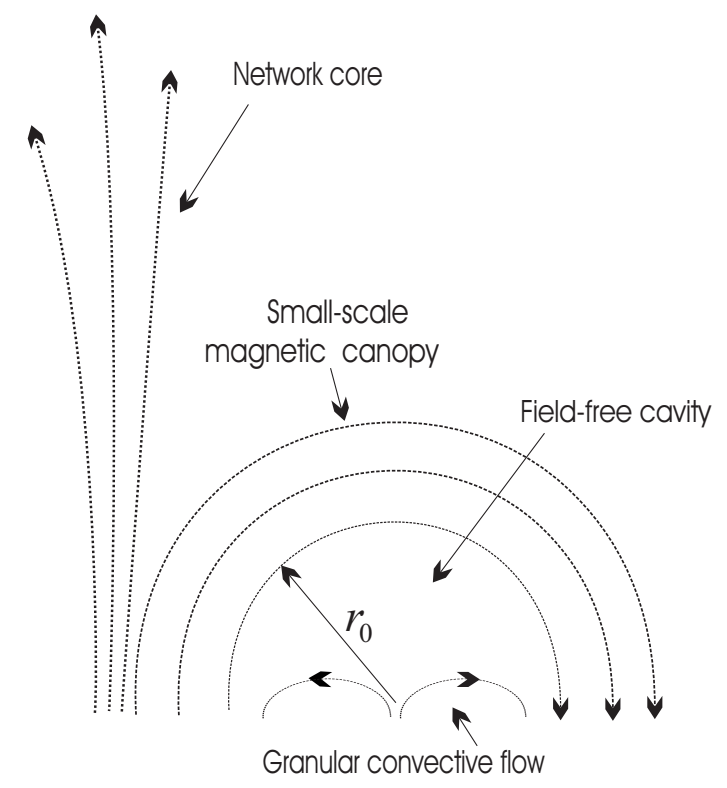

Fig. 1. Schematic picture of the magnetic canopy overlying a fieldfree cavity.

Here we use a model of small-scale bipolar magnetic canopy near a chromospheric network core and/or an active region. We suggest that granular cells may form field-free cylindrical cavities under the magnetic canopy due to the transport of magnetic flux towards boundaries. These cavities may trap high-frequency acoustic oscillations, while the lower-frequency harmonics may propagate upwards in form of magneto-acoustic waves.

Section 2 gives the analytical approach, obtained dispersion relation and resulting oscillation spectrum. Section 3 includes discussion and comparison of theoretical findings to observations. Section 4 briefly summarizes the results.

\section{The model}

We use the ideal magnetohydrodynamic (MHD) equations which can be written in the following form

$$
\begin{aligned}
& \frac{\partial \boldsymbol{B}}{\partial t}=\nabla \times(\boldsymbol{v} \times \boldsymbol{B}) \\
& \rho \frac{\partial \boldsymbol{v}}{\partial t}+\rho(\boldsymbol{v} \cdot \nabla) \boldsymbol{v}=-\nabla p+\frac{1}{4 \pi}(\nabla \times \boldsymbol{B}) \times \boldsymbol{B}, \\
& \frac{\partial \rho}{\partial t}+\nabla \cdot(\rho \boldsymbol{v})=0 \\
& \frac{\partial p}{\partial t}+(\boldsymbol{v} \cdot \nabla) p+\gamma p \nabla \cdot \boldsymbol{v}=0
\end{aligned}
$$

where $\boldsymbol{v}$ denotes the fluid velocity, $\boldsymbol{B}$ the magnetic field, $p$ the pressure, $\rho$ the mass density, and $\gamma$ the ratio of specific heats. Gravity effect is omitted from the consideration for the sake of simplicity. With this simple model we intend to estimate the potential of the proposed mechanism. Gravity will be taken into account in future developments of the present model.

We assume that the magnetic field is vertical in the core of the chromospheric network, but it gets the shape of smallscale closed loop systems in the surrounding. (McIntosh and Judge, 2001; Schrijver and Title, 2003). Granular motions then may form field-free cavities under the canopy as they carry the magnetic flux towards the cell boundaries. These field-free cavities have granular dimensions and may become resonators for acoustic oscillations. Consequently, we have two different regions (Fig. 1):

$\left\{\begin{array}{l}\text { region I - field-free cavity, } \quad r<r_{0}, \\ \text { region II - magnetic canopy, } r>r_{0} .\end{array}\right.$

We use a cylindrical coordinate system $(r, \phi, z)$ and consider an unperturbed cylindrical magnetic field $B_{0}$ in the canopy area. The magnetic field has only a $\phi$-component which depends on the distance $r$, i.e. $\boldsymbol{B}_{\mathbf{0}}=\left(0, B_{\phi}(r), 0\right)$. The equilibrium in the canopy is then satisfied if

$$
\frac{d}{d r}\left(p_{0}+\frac{B_{\phi}^{2}}{8 \pi}\right)+\frac{B_{\phi}^{2}}{4 \pi r}=0,
$$

where $p_{0}$ denotes the unperturbed pressure. To avoid further mathematical complications, we consider the unperturbed hydrodynamic pressure to be homogeneous. The equilibrium magnetic field is then current-free expressed as follows (Díaz et al., 2006)

$B_{\phi}=B_{\phi 0} \frac{r_{0}}{r}$.

Equations (1-4) are linearized, which yields

$\frac{\partial \boldsymbol{b}}{\partial t}=\nabla \times\left(\boldsymbol{u} \times \boldsymbol{B}_{\mathbf{0}}\right)$,

$\rho_{0} \frac{\partial \boldsymbol{u}}{\partial t}=-\nabla\left(p_{1}+\frac{1}{4 \pi} \boldsymbol{B}_{0} \cdot \boldsymbol{b}\right)+\frac{1}{4 \pi}\left(\boldsymbol{B}_{\mathbf{0}} \nabla\right) \boldsymbol{b}+$

$+\frac{1}{4 \pi}(\boldsymbol{b} \nabla) \boldsymbol{B}_{\mathbf{0}}$,

$\frac{\partial \rho_{1}}{\partial t}+\rho_{0} \nabla \cdot \boldsymbol{u}=0$,

$p_{1}=c_{0}^{2} \rho_{1}$,

where $\boldsymbol{b}, \boldsymbol{u}, p_{1}$ and $\rho_{1}$ are the perturbations of magnetic field, velocity, pressure and mass density, respectively, while $\rho_{0}$ is a uniform unperturbed mass density and $c_{0}=\left(\gamma p_{0} / \rho_{0}\right)^{1 / 2}$ corresponds to the homogeneous sound speed. Note, that a background flow is absent in our consideration.

For simplicity, we consider the 2-D case and restrict the analysis to the $(r, \phi)$-plane. In principle, the $z$-direction 
can also be considered, but this further complicates the presentation (namely, resonant absorption may take place) and it is left for future considerations. Then $r$ - and $\phi$-components of Eqs. (6-9) are given by

$\frac{\partial b_{r}}{\partial t}=\frac{B_{\phi}}{r} \frac{\partial u_{r}}{\partial \phi}$

$\frac{\partial b_{\phi}}{\partial t}=-B_{\phi} \frac{\partial u_{r}}{\partial r}+B_{\phi} \frac{u_{r}}{r}$,

$\rho_{0} \frac{\partial u_{r}}{\partial t}=-c_{0}^{2} \frac{\partial \rho_{1}}{\partial r}-\frac{B_{\phi}}{4 \pi} \frac{\partial b_{\phi}}{\partial r}+\frac{B_{\phi}}{4 \pi r} \frac{\partial b_{r}}{\partial \phi}-\frac{B_{\phi}}{4 \pi r} b_{\phi}$,

$\rho_{0} \frac{\partial u_{\phi}}{\partial t}=-\frac{c_{0}^{2}}{r} \frac{\partial \rho_{1}}{\partial \phi}$,

$\frac{\partial \rho_{1}}{\partial t}+\rho_{0} \frac{\partial u_{r}}{\partial r}+\rho_{0} \frac{u_{r}}{r}+\frac{\rho_{0}}{r} \frac{\partial u_{\phi}}{\partial \phi}=0$.

To get the oscillation spectrum in the cavity area, we have to solve the equations in the cavity and canopy regions separately and then merge the obtained solutions at the interface $\left(r=r_{0}\right)$.

Medium is field-free in the cavity $\left(r<r_{0}\right)$ and therefore can be described by pure hydrodynamics. Then Eqs. (1214) lead to the Bessel equation after Fourier analysis with respect to both the $t$ (time) and $\phi$ coordinates (the magnetic field is set to zero):

$$
\frac{\partial^{2} \rho_{1}}{\partial r^{2}}+\frac{1}{r} \frac{\partial \rho_{1}}{\partial r}+\left[\frac{\omega^{2}}{c_{0}^{2}}-\frac{m^{2}}{r^{2}}\right] \rho_{1}=0,
$$

where $\omega$ is the wave frequency, and $m$ is the azimuthal wave number.

In the magnetized canopy region $\left(r>r_{0}\right)$, Eqs. (10-14) lead to the Hain-Lust equation

$$
\begin{aligned}
& \frac{\partial}{\partial r}\left(\frac{\omega^{2} c_{0}^{2}}{\omega^{2}-m^{2} c_{0}^{2} / r^{2}}+v_{A}^{2}\right) \frac{\partial \hat{u}_{r}}{\partial r}+ \\
& \quad+\frac{\partial}{\partial r}\left(\frac{\omega^{2} c_{0}^{2}}{\omega^{2}-m^{2} c_{0}^{2} / r^{2}}+v_{A}^{2}\right) \frac{\hat{u}_{r}}{r}+ \\
& \quad+\left[\omega^{2}+\frac{4 v_{A}^{2}}{r^{2}}-\frac{m^{2} v_{A}^{2}}{r^{2}}\right] \hat{u}_{r}=0,
\end{aligned}
$$

where $v_{A}=B_{\phi} / \sqrt{4 \pi \rho_{0}}$ denotes the Alfvén speed.

Analytical solution of Eq. (16) is complicated. Therefore, for simplicity, we suppose that the magnetic energy is much higher than the hydrodynamic one within the canopy area. Consequently, we use the zero $\beta$ approximation hereinafter. The unperturbed configuration must be in equilibrium, therefore the unperturbed hydrodynamic pressure of the cavity and the magnetic pressure of the canopy must be balanced at the interface $\left(r=r_{0}\right)$

$\frac{c_{01}^{2} \rho_{01}}{\gamma}=\frac{B_{\varphi}^{2}\left(r_{0}\right)}{8 \pi}$ where

$c_{01}=\sqrt{\frac{\gamma p_{0}}{\rho_{01}}}$,

and $c_{01}$ and $\rho_{01}$ are the sound speed and the plasma mass density in the cavity. Equation (17) then gives the relation between the sound and Alfvén speeds at the interface

$c_{01}=\sqrt{\frac{\gamma}{2} \frac{\rho_{02}}{\rho_{01}}} v_{A 2}$,

where

$v_{A 2}=\frac{B_{\varphi}\left(r_{0}\right)}{\sqrt{4 \pi \rho_{02}}}$

is the Alfvén speed at the interface and $\rho_{02}$ is the plasma mass density in the canopy.

\subsection{Analytical solutions}

In the field-free cavity area under the canopy (region I), there are only acoustic waves. The $r$-dependence of the mass density perturbations in the acoustic waves is governed by Eq. (15) which has the general solution

$\rho_{1}=c_{1} J_{m}\left(k_{1} r\right)+c_{2} Y_{m}\left(k_{1} r\right)$,

where $J_{m}\left(k_{1} r\right)\left(Y_{m}\left(k_{1} r\right)\right)$ is the bessel function of the first (second) kind,

$k_{1}=\frac{\omega}{c_{01}}$

and $c_{1}, c_{2}$ are arbitrary constants. The perturbation must be finite at $r=0$. Therefore, $c_{2}=0$ and only the first term on the right hand side of expression (21) is non-vanishing.

The radial velocity component of the acoustic wave in the region I can be obtained using Eqs. (12) and (21) as (setting the magnetic field to be zero)

$\hat{u}_{r 1}=\frac{i c_{01}^{2}}{\rho_{01} \omega} c_{1} J_{m}^{\prime}\left(k_{1} r\right)$,

where the prime denotes the derivation with respect to $r$.

In the magnetic canopy (region II), where the cold plasma approximation is used, Eq. (16) gives

$\frac{\partial^{2} \hat{u}_{r}}{\partial r^{2}}-\frac{1}{r} \frac{\partial \hat{u}_{r}}{\partial r}+\left[\frac{\omega^{2}}{v_{A}^{2}(r)}+\frac{1}{r^{2}}-\frac{m^{2}}{r^{2}}\right] \hat{u}_{r}=0$.

This equation can be rewritten as

$\frac{1}{r} \frac{\partial}{\partial r}\left(r \frac{\partial \psi}{\partial r}\right)+\left[\frac{\omega^{2}}{v_{A}^{2}(r)}-\frac{m^{2}}{r^{2}}\right] \psi=0$,

where

$\hat{u}_{r}=r \psi, v_{A}^{2}(r)=\frac{B_{\phi 0}^{2}}{4 \pi \rho_{02}} \frac{r_{0}^{2}}{r^{2}}$. 

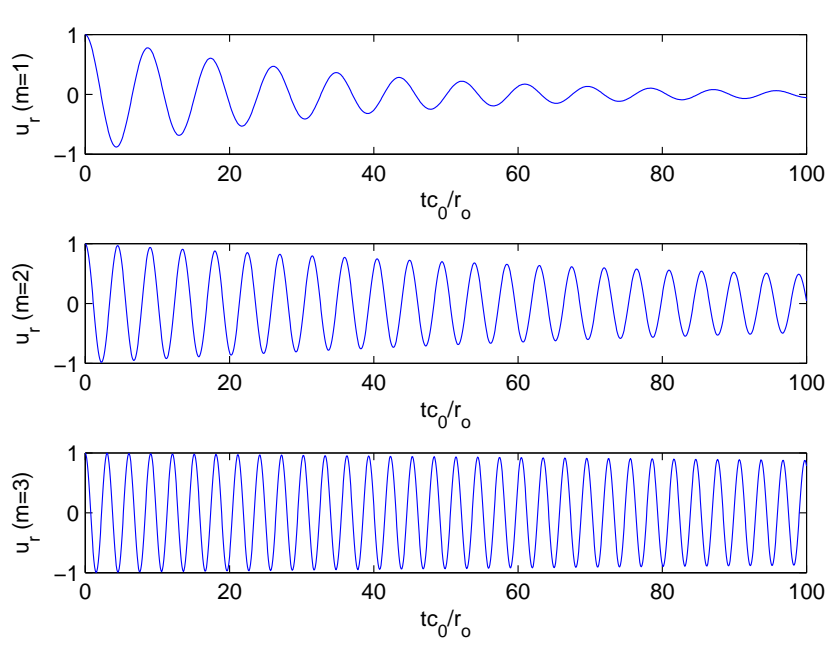

Fig. 2. Temporal dynamics of velocity perturbations in the field-free cavity areas for $m=1,2,3$ harmonics.

The solutions of this equation are the Bessel functions of half integer order (Abramowitz and Stegun, 1967; Díaz et al., 2006). In region II, only the outgoing wave is physically appropriate. Therefore, we choose the Hankel function

$\hat{u}_{r 2}=i c_{3} r H_{m / 2}\left(k_{2} r\right)$,

where $c_{3}$ is an arbitrary constant and

$k_{2}=\frac{\omega}{2 v_{A 2}}$.

The total pressure perturbations in the regions I and II are, respectively,

$p_{1}=c_{01}^{2} \rho_{1}$,

and

$\frac{B_{\phi} b_{\phi}}{4 \pi}=\frac{i \rho_{02} v_{A 2}^{2}}{\omega}\left[\frac{d \hat{u}_{r 2}}{d r}-\frac{\hat{u}_{r 2}}{r}\right]$.

Thus, the expressions (23), (26), (28), and (29) give the transverse velocity and the total pressure perturbations in the considered two regions.

\subsection{Dispersion relation}

The continuity of the velocity and the total pressure perturbations at the interface $\left(r=r_{0}\right)$ leads to

$c_{01}^{2} \rho_{1}=\frac{i \rho_{02} v_{A 2}^{2}}{\omega}\left[\frac{d \hat{u}_{r 2}}{d r}-\frac{\hat{u}_{r 2}}{r}\right]$,

$\hat{u}_{r 1}=\hat{u}_{r 2}$.

The substitution of the expressions $\rho_{1}, \hat{u}_{r 1}, \hat{u}_{r 2}$ into Eqs. (30) and (31) then gives

$c_{01}^{2} c_{1} J_{m}\left(k_{1} r_{0}\right)=-\frac{\rho_{02} v_{A 2}^{2}}{\omega} c_{3} r_{o} k_{2} H_{m / 2}^{\prime}\left(k_{2} r_{0}\right)$, $\frac{c_{01}^{2}}{\omega \rho_{01}} c_{1} k_{1} J_{m}^{\prime}\left(k_{1} r_{0}\right)=c_{3} r_{o} H_{m / 2}\left(k_{2} r_{0}\right)$.

The condition for a non-trivial solution of Eqs. (32-33), then yields the general dispersion relation, viz.

$\frac{\omega^{2}}{v_{A 2}^{2}} \frac{\rho_{01}}{\rho_{02}} \frac{J_{m}\left(k_{1} r_{0}\right)}{k_{1} J_{m}^{\prime}\left(k_{1} r_{0}\right)}=-\frac{k_{2} H_{m / 2}^{\prime}\left(k_{2} r_{0}\right)}{H_{m / 2}\left(k_{2} r_{0}\right)}$.

The dispersion relation (34) is a transcendental equation for the complex $\omega$. The imaginary part of wave frequency $\omega$ indicates a wave leakage from the field-free cavity into the ambient magnetic canopy. The question then arises how important this wave leakage is and which are the most sensitive parameters for trapping (or leakage) of the waves under the canopy. The analytical solution of Eq. (34) is complicated. Therefore, we apply numerical techniques to solve it.

The properties of the dispersion relation (34) depend on the azimuthal wave number $m$ and the ratio between sound $\left(c_{01}\right)$ and Alfvén $\left(v_{A 2}\right)$ speeds (or the ratio between $\rho_{01}$ and $\rho_{02}$, see Eq. 19). The dimension of the field-free cavity, $r_{0}$, also stands as a free parameter, but it influences only the wave periods.

Numerical solution of Eq. (34) shows that the ratio of imaginary $\omega_{i}$ and real $\omega_{r}$ parts significantly depends on the azimuthal wave number $m$. The ratio is higher for $m=1$ and quickly decreases with increasing $m$. Figure 2 shows the temporal dynamics of radial velocity perturbations in the field-free cavity region for modes corresponding to different $m$-values. The amplitude of the first $(m=1)$ harmonic quickly decreases, which indicates the rapid radiation of this mode into the overlying canopy. On the other hand, the $m=2$ mode undergoes a very small leakage and the $m=3$ mode has almost no leakage. Thus, the first harmonic of the acoustic oscillations cannot be trapped in the cavity area due to the rapid leakage into the canopy, while the higher harmonics, i.e. those with $m \geq 2$, can be easily trapped, which may lead to the observed increased acoustic power.

Figure 3 shows the ratio of the imaginary $\omega_{i}$ and real $\omega_{r}$ parts vs the ratio of the Alfvén and sound speeds for different harmonics. We see that the decrease of the ratio between the Alfvén and sound speeds leads to an enhanced leakage.

Figure 4 shows the dependence of real (top panel) and imaginary (bottom panel) parts of the frequency on the size $r_{0}$ of the field-free cavity region under the canopy. It is evident that the real part of the frequency decreases with increasing $r_{0}$, as can be expected from physical considerations.

\section{Discussion}

We have studied the spectrum of acoustic oscillations in a cylindrical field-free cavity under a small-scale bipolar magnetic canopy in the solar atmosphere. It is shown that the $m=1$ (where $m$ is the azimuthal wave number) harmonic 

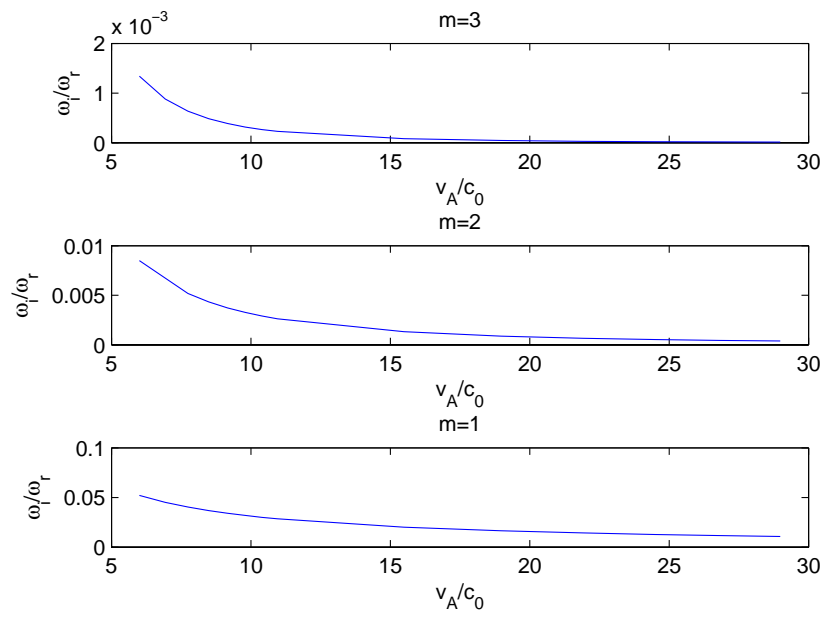

Fig. 3. The ratio of imaginary $\omega_{i}$ and real $\omega_{r}$ parts of wave frequency vs the ratio of Alfvén and sound speeds for $m=1,2,3$ harmonics.

of the acoustic oscillations cannot be trapped in the cavity as a result of the energy leakage in the upward direction. The energy radiation occurs through the propagation of fast magneto-acoustic waves in overlying magnetic canopy. However, higher $m \geq 2$ harmonics can be trapped in the cavity, leading to the observed increased high-frequency power in the photosphere.

There are three different explanations of power halos proposed in the literature: (i) the enhancement of acoustic emission by some unknown source (Braun et al., 1992; Brown et al., 1992; Jain and Haber, 2002), (ii) incompressible oscillations, such as Alfvén waves or transverse kink waves, in magnetic tubes (Hindman and Brown, 1998), and (iii) the interaction of acoustic waves with the overlying magnetic canopy (Muglach et al., 2005).

We suggest that the surroundings of magnetic network cores and active regions consist of many small-scale closed magnetic canopy structures (McIntosh and Judge, 2001; Schrijver and Title, 2003). Granular motions transport the magnetic field at boundaries and consequently create fieldfree cylindrical cavity areas under the canopy (see Fig. 1). These field-free cavities may be filled by trapped acoustic oscillations stochastically excited by granular motions (Lighthill, 1952; Selwa et al., 2004). For a typical photospheric sound speed of $\sim 7 \mathrm{~km} / \mathrm{s}$ and a typical granular radius of $\sim 400 \mathrm{~km}$, the period of the $m=2$ harmonic is $\sim 3 \mathrm{~min}$ and the period of the $m=3$ harmonic is $\sim 2 \mathrm{~min}$. The range of observed enhanced power frequency is about $5-7 \mathrm{mHz}$ (with periods of 2-3 min), which is in agreement with our findings. The $m=1$ harmonic is leaky and, therefore, can not be trapped under the canopy.

If power halos are related to the acoustic waves then observations should show an enhancement in continuum intensity
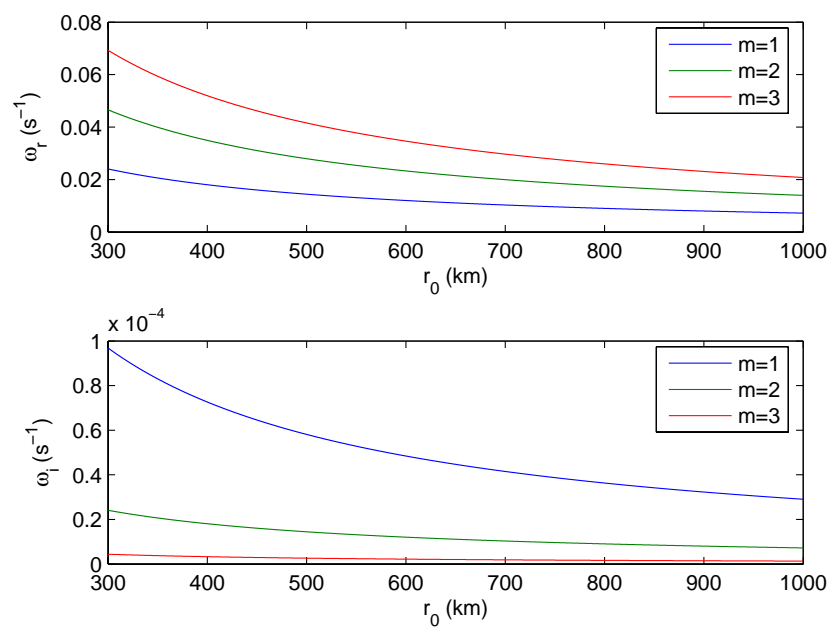

Fig. 4. Real $\omega_{r}$ (upper panel) and imaginary $\omega_{i}$ (lower panel) parts of wave frequency vs the radius of field-free cavity $r_{0}$ for $m=1,2,3$ harmonics

power maps as well, but in opposite observations show a lack of power halos in the maps (Hindman and Brown, 1998; Jain and Haber, 2002; Muglach et al., 2005), what needs an adequate explanation. Here, we suggest a natural explanation for this phenomenon. Indeed, the antinodes of mass density and velocity components in standing acoustic oscillations are located at different places: the location of the maximal velocity oscillations corresponds to the location of the zero amplitude intensity oscillations and vice versa. Therefore, the enhanced Doppler velocity power at fixed height of the atmosphere automatically suggest the absence of intensity power at the same height. This suggestion can be checked by searching intensity power halos at different heights from the surface. We believe that new observations with high resolution from the "Hinode" spacecraft will shed light on this problem.

However, the formation of power halos due to incompressible oscillations can not be completely ruled out. Recent theoretical and two-dimensional numerical simulations outlined the importance of the $\beta \sim 1$ region in the solar atmosphere, where wave conversion or reflection occurs (Rosenthal et al., 2002; Bogdan et al., 2003; Zaqarashvili and Roberts, 2006; Kuridze and Zaqarashvili, 2007). Indeed, our consideration implies that the hydrodynamic and magnetic pressures have approximately the same value at the interface (see Eq. 17). Therefore, the acoustic waves may transfer energy into the incompressible waves through non-linear interactions (Zaqarashvili and Roberts, 2006; Kuridze and Zaqarashvili, 2007), which may lead to the observed power halos. However, the observed oscillations hardly show nonlinear behavior, which complicates the explanation of power halos by incompressible oscillations considerably. 
Another important alternative for the proposed mechanism can be resonant absorption of waves in inhomogeneous plasma (Tirry et al., 1998; Pintér and Goossens, 1999; Pintér et al., 2007). This process can also be particularly important for 3-D consideration of proposed model, when one considers the wave propagation in the z-direction as well. This is out of the scope of present paper, but would be interesting to study in the future.

It must be mentioned that the equilibrium used in this paper is simplified as gravitational stratification, which is important in the solar atmosphere, is ignored. The stratification leads to a Klein-Gordon equation for the propagating waves with a cut-off for wave frequencies (Roberts, 2004; Erdélyi et al., 2007). However, wave propagation in inclined magnetic field may lead to decrease of cut-off frequencies and simplifies the penetration of lower-frequency oscillations in higher regions (De Pontieu et al., 2004). Non of the harmonics has purely vertical propagation in our model because of the non-zero azimuthal wave number $m$; all harmonics propagate with an angle to the vertical (note, that all harmonics have standing wave behavior along azimuthal direction). Therefore their frequencies are above the cut-off value and consequently the harmonics are not evanescent. However, inclusion of gravitational stratification is necessary for a more profound understanding of the wave trapping in cavities. This will be the next step of our study.

\section{Conclusion}

We have studied the spectrum of acoustic oscillations in the cylindrical field-free cavity regions under the small-scale magnetic canopy near the magnetic network cores and active regions. We found that the first harmonic of acoustic oscillations cannot be trapped in the cavity due to the energy radiation by fast magneto-acoustic waves in the canopy. However, the higher $(m \geq 2)$ harmonics can be trapped there, leading to the observed enhancement of high-frequency acoustic power in the photosphere. Future detailed study of the proposed mechanism including gravitational stratification and 3$\mathrm{D}$ models is necessary.

Acknowledgements. The work was supported by the grant of Georgian National Science Foundation GNSF/ST06/4-098. The work of B. M. Shergelashvili has been supported by K. U. Leuven scholarship - PDM/06/116. These results were obtained in the framework of the projects GOA/2004/01 (K. U. Leuven), G.0304.07 (FWOVlaanderen) and C 90203 (ESA Prodex 8). Financial support by the European Commission through the SOLAIRE Network (MTRNCT-2006-035484) is gratefully acknowledged.

Topical Editor R. Forsyth thanks two anonymous referees for their help in evaluating this paper.

\section{References}

Abramowitz, M. and Stegun I. A.: Handbook of Mathrmatical Functions (Dover), 1967

Bogdan, T. J., Hansteen, M., Carlsson, V., et al.: Waves in the magnetized solar atmosphere. II. Waves from localized sources in magnetic flux concentrations, Astrophys. J., 599, 626-660, 2003.

Braun, D. C., Lindsey, C., Fan, Y., and Jefferies, S. M.: Local acoustic diagnostics of the solar interior, Astrophys. J., 392, 739-745, 1992.

Brown, T. M, Bogdan, T. J., Lites, B. W., and Thomas, J. H.: Localized sources of propagating acoustic waves in the solar photosphere, Astrophys. J., 394, 65-68, 1992.

Centeno, R., Socas-Navarro, H., Lites, B., Kubo, M., Frank, Z., Shine, R., Tarbell, T., Title, A., Ichimoto, K., Tsuneta, S., Katsukawa, Y., Suematsu, Y., Shimizu, T., and Nagata, S.: Emergence of Small-Scale Magnetic Loops in the Quiet-Sun Internetwork, Astrophys. J., 666, 137-140, 2007.

De Pontieu, B., Erdelyi, R., and James, E. S. P.: Solar chromospheric spicules from the leakage of photospheric oscillations and flows, Nature, 430, 536-539, 2004.

De Wijn, A. G., Rutten, R. J., Haverkamp, E. M. W. P., and Sutterlin, P.: DOT tomography of the solar atmosphere. IV. Magnetic patches in internetwork areas, Astron. Astrophys., 441, 11831190, 2005.

Díaz, A. J., Zaqarashvili, T., and Roberts, B.: Fast magnetohydrodynamic oscillations in a force-free line-tied coronal arcade, Astron. Astrophys., 455, 709-717, 2006.

Erdelyi, R., Malins, C., Toth, G., and de Pontieu, B.: Leakage of photospheric acoustic waves into non-magnetic solar atmosphere, Astron. Astrophys., 477, 1299-1311, 2007.

Evans, D. J. and Roberts, B.: The influence of a chromospheric magnetic field on the solar p- and f-modes. II - Uniform chromospheric field, Astrophys. J., 356, 704-719, 1990.

Hindman, B. W. and Brown, T. M.: Acoustic power maps of solar active regions, Astrophys. J., 504, 1029-1034, 1998.

Jain, R. and Haber, D.: Solar p-modes and surface magnetic fields: Is there an acoustic emission?, MDI/SOHO observations, Astron. Astrophys., 387, 1092-1099, 2002.

Krijger, J. M., Rutten, R. J., Lites, B. W., Straus, Th., Shine, R. A., and Tarbell, T. D.: Dynamics of the solar chromosphere. III. Ultraviolet brightness oscillations from TRACE, Astron. Astrophys., 379, 1052-1082, 2001.

Kuridze, D. and Zaqarashvili, T. V.: Resonant energy conversion of 3-minute intensity oscillations into Alfven waves in the solar atmosphere, J. Atmos. Solar Terr. Phys., 70, 351-355, 2007.

Lighthill, M. J.: On sound generated aerodynamically. I. General theory, Proc. Roy. Sos., 211, 564-587, 1952.

McIntosh, S. W. and Judge, P. G.: On the Nature of Magnetic Shadows in the Solar Chromosphere, Astrophys. J., 561, 420-426, 2001.

Muglach, K.: Dynamics of solar active regions. I. Photospheric and chromospheric oscillations observed with TRACE, Astron. Astrophys., 401, 685-697, 2003.

Muglach, K., Hofmann, A., and Staude, J.: Dynamics of solar active regions. II. Oscillations observed with MDI and their relation to the magnetic field topology, Astron. Astrophys., 437, 10551060, 2005.

Pintér, B., Erdelyi, R., and Goossens, M.: Global oscillations in a magnetic solar model. II. Oblique propagation, Astron. Astro- 
phys., 347, 321-334, 1999.

Pintér, B. and Goossens, M.: Oscillations in a magnetic solar model. I. Parallel propagation in a chromospheric and coronal magnetic field with constant Alfven speed, Astron. Astrophys., 466, 377388, 2007.

Roberts, B.: MHD Waves in the Solar Atmosphere, in: Proc. SOHO 13 - Waves, Oscillations and Small-Scale Transient Events in the Solar Atmosphere: A Joint View from SOHO and TRAC', Palma de Mallorca, Balearic Islands, Spain (ESA SP-547), 1, 2004.

Rosenthal, C. S., Bogdan, T. J., Carlsson, M., Dorch, S. B. F., Hansteen, V., McIntosh, S. W., McMurry, A., Nordlund, Å, and Stein, R. F.: Waves in the magnetized solar atmosphere. I. Basic processes and internetwork oscillations, Astrophys. J., 564, 508-524, 2002

Selwa, M., Skartlien, R., and Murawski, K.: Numerical simulations of stochastically excited sound waves in a random medium, Astron. Astrophys., 420, 1123-1127, 2004.
Shergelashvili, B. M. and Poedts, S.: On the effect of the inhomogeneous subsurface flows on the high degree solar p-modes, Astron. Astrophys., 438, 1083-1097, 2005

Schrijver, C. J. and Title, A. M.: The Magnetic Connection between the Solar Photosphere and the Corona, Astrophys. J., 597, L165L168, 2003.

Tirry, W. J., Goossens, M., Pinter, B., Cadez, V., and Vanlommel, P.: Resonant Damping of Solar p-Modes by the Chromospheric Magnetic Field, Astrophys. J., 503, 422-428, 1998.

Vecchio, A., Cauzzi, G., Reardon, K. P., Janssen, K., and Rimmele, T.: Solar atmospheric oscillations and the chromospheric magnetic topology, Astron. Astrophys., 461, 1-4, 2007.

Zaqarashvili, T. V. and Roberts, B.: Two-wave interaction in ideal magnetohydrodynamics, Astron. Astrophys., 452, 1053-1058, 2006. 